\title{
DNA Mismatch Repair State And Clinical Pathological Characteristics Of Ovarian Clear Cell Carcinoma
}

Qian Liu

Peking Union Medical College Hospital

Huimei Zhou ( $\square$ mayflower0808@126.com )

Jiaxin Yang

Peking Union Medical College Hospital

Xiaohua Shi

Peking Union Medical College Hospital

\section{Research}

Keywords: Ovarian clear cell carcinoma, DNA mismatch repair, Prognosis

Posted Date: July 29th, 2020

DOI: https://doi.org/10.21203/rs.3.rs-47570/v1

License: (c) (1) This work is licensed under a Creative Commons Attribution 4.0 International License. Read Full License 


\section{Abstract}

Background: Ovarian clear cell carcinoma (OCCC) is a distinct subtype of epithelial ovarian cancer. With the exception of very early stage disease, OCCCs are associated with worse prognosis due to poor response to platinum-based chemotherapy compared with serous carcinoma. OCCCs with microsatellite instability (MSI) may represent a unique subset and may open an alternative therapeutic avenue for a fraction of patients with OCCC. The purpose of this study is to explore the role of DNA mismatch repair (MMR) in the genesis and development of clear cell carcinoma by determining the differential expression of MMR gene in different subtypes of ovarian carcinoma and the expression of MMR protein in clear cell carcinoma, so as to provide ideas for future tumor treatment.

Results: The expression of MSH6 gene in HGSC group was higher than that in OCCC group, and the difference had statistical significance $(P=0.01)$. The expression of MLH1 gene in OCCC group was higher than that in HGSC group, and the difference had statistical significance $(P=0.0221)$. There was no significant difference in the expression of $\mathrm{MSH} 2$ gene between the two groups. There were no significant differences in the expression of the three genes between OCCC group with good prognosis (group $\mathrm{G}$ ) and OCCC group with poor prognosis (group P). In order to further verify the expression of MMR protein in OCCC group, immunohistochemistry was performed, and the results showed that $92.3 \%$ of the patients $(36 / 39)$ had pMMR, $7.69 \%$ of the patients (3/39) had dMMR. The average follow-up time of patients with dMMR was 19.3 months, and there was no recurrence or death.

Conclusion: There were differences in the expression of MSH6 and MLH1 genes between ovarian clear cell carcinoma and high-grade serous carcinoma, indicating that the two subtypes of tumor may have different origins. The prognosis of clear cell carcinoma patients with dMMR was better as of the followup date, suggesting that dMMR may be an early event of clear cell carcinoma.

\section{Background}

Epithelial ovarian cancer (EOC) is a type of gynecological malignant tumor which has the highest mortality. About $61 \%$ of the patients were diagnosed as advanced when they first visit the hospital, and the 5 -year survival rate was only about $27 \%{ }^{[1]}$. Ovarian clear cell carcinoma (OCCC) accounts for $10 \%$ of epithelial ovarian cancer. Both the biological behavior and clinical characteristics of ovarian clear cell carcinoma are different from those of other types of epithelial ovarian cancers, often showing chemotherapy resistance and has poor prognosis ${ }^{[2]}$. The chemotherapy efficacy of paclitaxel combined with carboplatin is low, about $22-56 \%{ }^{[3-5]}$. Therefore, searching for new tumor markers and therapeutic targets has always been a hot topic in the field of clear cell carcinoma. OCCCs with microsatellite instability (MSI) may represent a unique subset and may open an alternative therapeutic avenue for a fraction of patients with OCCC. The purpose of this study is to explore the role of MMR in the genesis and development of clear cell carcinoma by determining the differential expression of MMR gene in different subtypes of ovarian carcinoma and the expression of MMR protein in clear cell carcinoma, so as to provide ideas for future tumor treatment. 


\section{Patients And Methods}

\section{Inclusion and exclusion criteria}

Inclusion criteria and exclusion criteria: inclusion criteria: (1) the primary lesion occurred in the ovary; (2) ovarian cancer staging surgery, tumor cell reduction surgery or laparoscopic biopsy was performed; (3) The pathological diagnosis was clear and the pathological specimens were well preserved; (4) FIGO stage was IIIV; (5) the follow-up data were complete; (6) no radiotherapy, chemotherapy and other anti-tumor treatment were received before surgery.

\section{Clinical data}

1) Group for genetic test: 36 patients who in accordance with the inclusion criteria were enrolled in Beijing Union Medical College Hospital from November 19, 2012 to November 18, 2016, including 24 cases of clear cell carcinoma and 12 cases of high-grade serous carcinoma. All patients were treated by cytoreductive surgery/ovarian cancer staging, and platinum based first-line chemotherapy was performed.

2) Group for Immunohistochemistry: 39 patients who in accordance with the inclusion criteria were enrolled in Beijing Union Medical College Hospital from January 7, 2015 to April 25, 2018. All of the 39 cases were all clear cell carcinoma. All patients were treated by cytoreductive surgery/ovarian cancer staging, and platinum based first-line chemotherapy was performed.

\section{NanoString expression analysis}

The differential expression level of genes in tumor tissue samples of the patients were analyzed. The requirements of histologic specimen of the selected cases are: effective area $>1.5^{\star} 1.5 \mathrm{~cm}, 3-5$ wax rolls with the thickness of 10um, and the number of wax roll increased according to the tissue area of the puncture specimen. The experimental process includes: total RNA extraction, quality control of samples, overnight hybridization, elution and purification of hybrid products, sample plate preparation, sample plate scanning, and results output.

\section{Immunohistochemistry}

Immunohistochemistry was performed to detect the expression of MLH1, MSH2, MSH6 and PMS2 in tumor tissues: paraffin blocks with samples were taken from tumor tissues and paracancerous tissues of patients, and the tumor cell rich areas (> 50\% were tumor cells) and paracancerous tissues were stained with immunohistochemistry. Among them, the ready-to-use MLH1 mouse anti-human monoclonal antibody (ESO5), the ready-to-use MSH2 mouse anti-human monoclonal antibody (FE11), the ready-touse MSH6 mouse anti-human monoclonal antibody (EP49) and the ready-to-use PMS2 mouse antihuman monoclonal antibody (EP51) were purchased from Zhongshan Company (Beijing). The experiment was performed according to the instructions, the sections were dewaxed and repaired by high pressure thermal repair with $1 \mathrm{mmol} / \mathrm{L}$ EDTA antigen repair solution (PH8.0). The sections were incubated 
with $30 \mathrm{ml} / \mathrm{L} \mathrm{H}_{2} \mathrm{O}_{2}$ for 10 min and washed by the distilled water. Then soaked by phosphate buffered saline (PBS) for $5 \mathrm{~min}$. The sections were incubated with normal rabbit serum working solution for $10 \mathrm{~min}$, and then incubated with primary antibodies of MLH1, MSH2, MSH6 and PMS at $37^{\circ} \mathrm{C}$ for $2 \mathrm{~h}$, washed with PBS for 3 times, $3 \mathrm{~min}$ each time. After that, the sections were incubated with the secondary antibody for $15 \mathrm{~min}$, rinsed with PBS for 3 times, $3 \mathrm{~min}$ for each time. Then the sections were incubated with the third antibody for $15 \mathrm{ml}$, and washed with PBS for 3 times, 3 min for each time. At last, diaminobenzidine (DAB) was used for developing, and hematoxylin was used for counterstaining. Then Dehydration, transparent, and sealing was performed for each section. PBS was used as the negative control of primary antibody, and the expression of paracancerous tissue was considered as positive control.

\section{Results determination}

MLH1, MSH2, MSH6 and PMS2 were all located in the nucleus of tumor cells, with yellow or brown granules as the expression patterns (+), and the nucleus of tumor cells without staining were considered as the deletion (-). The absence of any one type of MLH1, MSH2, MSH6 and PMS2 protein (-) was determined as defective DNA mismatch repair(dMMR), while the expression of all of the MLH1, MSH2, MSH6 and PMS2 proteins were determined as proficient DNA mismatch repair (pMMR) ${ }^{[6,7]}$. The evaluation process was independently completed by two senior pathologist using double blind method, and the disputed cases were further submitted to the superior physician for interpretation.

\section{Follow up}

The follow-up was performed by outpatient reexamination and telephone follow-up. The follow-up lasted from the beginning of the surgery to January 2019. Progression free survival (PFS) was defined as the time from staging or cytoreductive surgery to tumor recurrence, metastasis or death caused by any reason. Overall survival (OS) was defined as the time from staging or cytoreductive surgery to death of patients for any reason.

\section{Statistical analysis}

SPSS 23.0 software was used for statistical analysis. Chi-square test was performed to compare the enumeration data, and Kaplan-Meier method was performed for survival analysis.

\section{Results}

1. 1) Ovarian cancer patients with different histological types were divided into two groups, high-grade serous carcinoma group (HGSC group) and ovarian clear cell cancer group (OCCC group). The general clinical characteristics of the patients in these two groups were shown in Table 1. 
Table 1

Clinical characteristics of the patients in two groups

\begin{tabular}{|c|c|c|}
\hline & HGSC & occc \\
\hline & $N=12$ & $N=24$ \\
\hline Age (mean) & 57.6 & 50.7 \\
\hline Staging & & \\
\hline I, II & 1 & 15 \\
\hline III, IV & 11 & 9 \\
\hline Primary treatment & & \\
\hline Surgery + chemotherapy & 12 & 23 \\
\hline Chemotherapy scheme & $\begin{array}{l}\text { TC/Taxotere + } \\
\text { Carboplatin/TP }\end{array}$ & $\begin{array}{l}\text { TC/Taxotere + Carboplatin /TP/T + } \\
\text { Oxaliplatin }\end{array}$ \\
\hline $\begin{array}{l}\text { Number of treatment courses } \\
\text { (average) }\end{array}$ & 7.25 & 5.74 \\
\hline Median recurrence time & 8.83 & 3.58 \\
\hline
\end{tabular}

2) Comparison of the prognosis of patients in the two groups. The mean follow-up time of patients in OCCC group was 57.05 months, with 12 cases recurred, and the average recurrence interval was 3.58 months. The mean follow-up time of patients in HGSC group was 34.77 months, with 12 cases recurred, the mean recurrence interval was 8.83 months, PFS of OCCC group was significantly shorter than that of HGSC group $(P=0.001)$, and the survival curve was shown in Fig. 1. The OS of OCCC group was shorter than that of HGSC group, but the difference was not statistically significant $(P=0.278)$, as shown in Fig. 2.

2) The differential expression levels of MSH2, MSH6 and MLH1 in HGSC and OCCC groups were detected by using NanoString method. The results indicated that the expression level of MSH6 in HGSC group was significantly higher than that in OCCC group $(P=0.01)$. The expression level of MLH1 in OCCC group was significantly higher than that in HGSC group $(P=0.0221)$. There was no difference in the expression of $\mathrm{MSH} 2$ between the two groups. Furthermore, the expression levels of MSH2, MSH6 and MLH1 in the two groups with good or poor prognosis in OCCC group were analyzed and the differences showed no statistical significance (Table 2). 
Table 2

mRNA expression in tumor tissues of patients in HGSC and OCCC group

\begin{tabular}{|c|c|c|c|c|c|c|}
\hline & HGSC & OCCC & & & & $\mathbf{P}$ \\
\hline & $n=12$ & $n=24$ & & & & \\
\hline & & $P(n=12)$ & $G(n=12)$ & Sum $(n=24)$ & $\mathrm{P*}$ & \\
\hline $\mathrm{MSH} 2$ & $563.4 \pm 171.7$ & $498.3 \pm 160.2$ & $510.9 \pm 90.8$ & $504.6 \pm 127.5$ & 0.782 & 0.25 \\
\hline MSH6 & $740.4 \pm 172.8$ & $584.0 \pm 151.6$ & $591.6 \pm 147.9$ & $587.8 \pm 146.5$ & 0.869 & 0.01 \\
\hline MLH1 & $455.3 \pm 105.2$ & $540.0 \pm 126.8$ & $529.8 \pm 88.0$ & $534.9 \pm 106.8$ & 0.84 & 0.0221 \\
\hline
\end{tabular}

2.1) A total of 39 cases of clear cell carcinoma were randomly enrolled, and their clinical characteristics were shown in Table 3.

Table 3

Basic clinical characteristics of patients with clear cell carcinoma

\begin{tabular}{|ll|}
\hline Clinical characteristics $(\mathbf{n}=\mathbf{3 9})$ & \\
\hline Age & 54.8 \\
\hline Average age & \\
\hline STAGE & 24 \\
\hline I, II & 15 \\
\hline III, IV & \\
\hline Primary treatment & 39 \\
\hline Surgery + chemotherapy & TC/PC/CC/ nedaplatin + docetaxel \\
\hline Primary chemotherapy scheme & 4.9 \\
\hline Number of treatment courses (average) & \\
\hline Follow-up & 15.6 \\
\hline Average follow-up time $(\mathrm{m})$ & $28.2 \%(11 / 39)$ \\
\hline Recurrence rate & $8.12(0-13.7)$ \\
\hline Average recurrence time after initial treatment $(\mathrm{m})$ & \\
\hline
\end{tabular}

2) MMR system 
The expression of MMR protein (MSH2, MSH6, MLH, PMS2) in tumor tissue and paracancerous tissue was detected by immunohistochemistry, and the results showed that the expression of MMR protein was absent in 3 patients, that was $7.69 \%$ (3/39) was dMMR and 92.3\% (36/39) was pMMR. The specific situation of the 3 patients was shown in Table 4.

Table 4

Basic information of patients with dMMR

\begin{tabular}{|llllllll|}
\hline & Age & Staging & Treatment & chemotherapy & Follow-up & recurrence & Loss of expression \\
\hline 1 & 50 & IIIA1 & CRS & TC*6 & 26 & No & PMS2 \\
2 & 47 & IIIA & CRS & TC*6 & 16 & No & MSH2 \\
3 & 83 & IIIC & CRS & Carboplatin *1 & 14 & No & PMS2 \\
\hline
\end{tabular}

There was no significant difference in age of onset between dMMR group and pMMR group $(P=0.328)$. The staging of $d M M R$ group was higher than that of pMMR group $(P=0.04)$. In terms of prognosis, the recurrence rate and mortality rate in the dMMR group were lower than those in the pMMR group. However, the difference showed no statistical significance (Fig. 3, Fig. 4).

\section{Discussion}

Ovarian cancer is one of the most common gynecological malignant tumors, and has the highest mortality. Every year, there are about 240,000 new cases and 150,000 deaths worldwide. The combination of surgery and chemotherapy improves the overall response rate and clinical remission rate of patients with ovarian cancer, and prolongs the median survival time, and the remission rate of patients with primary treatment could reach as high as $80 \%$. However, with the continuation of treatment, $70 \%$ of patients who had achieved complete clinical remission still have recurrence, and its survival rate has not been substantially improved in recent years ${ }^{[8]}$. At present, the treatment of ovarian cancer does not consider the type of tissue. However, ovarian cancer with different tissue types has different molecular and biological characteristics. With the development of molecular biology, it is gradually recognized that the molecular typing of tumor may be closely related to its clinical pathological characteristics, biological behavior, treatment and prognosis. Therefore, ovarian cancer is likely to be treated according to the tissue type or even molecular typing in the future.

Mismatch repair system (MMR) is a safety guarantee system of the body, which can maintain the integrity and stability of genetic materials. It mainly targets on the removal and repair of base mismatch produced in the process of DNA recombination and replication. It is a biochemical process involved in many proteins ${ }^{[9]}$ and also an important means for cells to correct replication errors, and often occurs in the process of proliferation to maintain gene accuracy. The basic function of mismatch repair is to remove the insertion/deletion loop caused by base mismatch and DNA polymerase slide in the process of replication, correct the recombination of non-homologous chromosomes and maintain the stability of the 
whole genome ${ }^{[10]}$. Frequent DNA replication errors can cause changes in the microsatellite sequence of cells, which is manifested by the length increase or decrease of microsatellite fragments, which is defined as microsatellite instability (MSI), a specific phenotype of mismatch repair system functional defects ${ }^{[11}$, ${ }^{12]}$. In patients with colorectal cancer, dMMR/MSI patients have unique clinical pathological features, including early onset, high incidence in the right hemicolon, mostly mucinous adenocarcinoma and poorly differentiated adenocarcinoma in histological types, presence of Crohn's like lymphocyte reaction, and good prognosis ${ }^{[13]}$. Recently, the application of PD-1 antibody pembrolizumab in solid cancer with microsatellite instability (MSI)-H or mismatch repair (MMR) deficient has been approved ${ }^{[14]}$. The research progress of MSI and the application of pembrolizumab and other drugs have provided new treatments for tumor therapy.

In gynecological tumors, the proportion of MSI in endometrial cancer is $15-30 \%[15,16]$. However, the mechanism of the inactivation of mismatch repair gene in ovarian cancer and its correlation with the stage, grade, classification, prognosis and drug resistance of ovarian cancer are still not clear, and there is no large data report yet. There are still many controversies about the correlation between microsatellite instability and clinical pathological subtypes of epithelial ovarian cancer. It was reported that the proportion of MSI in ovarian cancer was $2-20 \%$, which was lower than that in endometrial cancer ${ }^{[17-21]}$. Tangir et al. found that the incidence of MSI-H was very low in serous adenocarcinoma, but high in borderline epithelial tumors, about $27.8 \%-30 \%$. These all suggested that tumors with different histological types and different biological behaviors had different etiology ${ }^{[22]}$. However, Shih et al analyzed 31 cases of epithelial ovarian cancer with low malignant potential but MSI was not found, and Arzimanoglouet found that there was no significant correlation between MSI and pathological types of ovarian cancer ${ }^{[24]}$. This study compared high-grade serous carcinoma and clear cell carcinoma at the gene expression level, and found that there was difference in the expression of MHS6 and MLH1, suggesting that MMR may play a different role in the occurrence and development of the two subtypes of tumor, which still needed to be confirmed by large sample-sized prospective study.

Although microsatellite instability is not common in epithelial ovarian cancer, more and more studies have shown that the proportion of MSI in clear cell carcinoma is high. Kathy et al detected MSI-H in 6 of 42 cases of ovarian clear cell carcinoma ${ }^{[25]}$. Gras et al. found that many ovarian cancers with MSI also followed the same process of MLH-1 promoter methylation and coding for mononucleoside bundle mutation accumulation ${ }^{[26]}$. In this study, the proportion of MMR protein expression deficiency in 39 patients was $7.7 \%$. Patients with clear cell carcinoma were divided into two groups according to their prognosis, and the results showed that there was no difference in MMR gene expression between the two groups. Moreover, three patients were in stage III of surgical-pathologic staging. These indicated that genetic instability caused by mismatch repair system may participate in the malignant transformation of clear cell carcinoma, and play a promoting role in the initiation of tumor.

Many molecules and enzymes are involved in MMR system during DNA repair. MLH1 and MSH2 are two main participants, and they often form dimers with their homologous MMR proteins. MLH1 and PMS2, 
MSH2 and MSH6 form functional dimer complexes respectively. MLH1 and MSH2 are the dominant proteins of dimer, and PMS2 and MSH6 are the paired proteins. The mutations of MLH1 and MSH2 can cause the degradation of dimer complexes, resulting in the loss of expression of dominant protein and paired protein. If the expression of MLH1 protein is absent, the expression of PMS2 protein is concomitantly absent at the same time. Similarly, if the expression of MSH2 protein is absent, the expression of MSH6 protein is concomitantly absent at the same time. The loss of MLH1 and MSH2 expression is mutually exclusive, and only one protein can be absent ${ }^{[27,28]}$. In this study, there were only 3 patients had MMR protein expression loss, including 2 cases with PMS2 protein loss and 1 case with $\mathrm{MSH} 2$ protein loss. The probability of PMS2 expression loss was very high, and MLH1 expression loss was not detected in the cases with PMS2 expression loss, while MSH6 expression loss was not detected in the cases with MSH2 expression loss. Therefore, it is assumed that the patterns of DNA repair by proteins in clear cell carcinoma with dMMR may change, but it needs to be confirmed by further studies.

\section{Conclusions}

In conclusion, there are a few cases of MSI in ovarian cancer. At present, there are still controversies in these researches. This study suggested that there was difference in the expression of MMR gene between ovarian clear cell carcinoma and high-grade serous carcinoma, and the pattern of MMR protein deficiency in clear cell carcinoma was different from that reported in the literature. Our group is currently investigating the correlation between $\mathrm{MSI}$ and immune checkpoint in ovarian clear cell carcinoma. It is believed that the follow-up research may open the door for new treatment of ovarian clear cell carcinoma.

\section{Abbreviations}

EOC: Epithelial ovarian cancer ;OCCC : Ovarian clear cell carcinoma ; MSI: microsatellite instability; MMR: DNA mismatch repair ; dMMR :Defective DNA mismatch repair; pMMR : Proficient DNA mismatch repair ;PFS : Progression free survival ;OS : Overall survival ;HGSC group : High-grade serous carcinoma group ;OCCC group: Ovarian clear cell cancer group ;MSI :microsatellite instability

\section{Declarations}

\section{Acknowledgements}

We thank all patients for participating in the follow-up.

\section{Funding}

This study was funded by the Chinese Academy of Medical Sciences Initiative of Innovative Medicine(CAMS-2017-I2M-1-002).

\section{Availability of data and materials}


All data generated or analyzed during this study are included in the published article.

\section{Authors' contributions}

Qian Liu designed the study, performed the data analyses and wrote the manuscript.

Huimei Zhou designed the study, performed the data analyses,reviesed the manuscript and approved the final version of the manuscript.

Jiaxin Yang, Xiaohua Shi collected and checked the data and approved the final version of the manuscript.

\section{Ethics approval and consent to participate}

This study was approved by the Institutional Review Board of PUMCH.

\section{Consent for publication}

No applicable.

\section{Competing interests}

The authors declare that they have no competing interests.

\section{Publisher' Note}

Springer Nature remains neural with regard to jurisdictional claims in published map and institutional affiliations.

\section{Author details}

1 Department of Gynaecologic Oncology,Peking Union Medical College Hospital,Beijing,P eople's Republic of China

2 Department of Pathology,Peking Union Medical College Hospital,Beijing,People's Republic of China.

\section{References}

1. Chang SJ, et al. Role of aggressive surgical cytoreduction in advanced ovarian cancer. J Gynecol Oncol. 2015;26(4):336-42.

2. Tan DS, Kaye S. Ovarian clear cell adenocarcinoma: a continuing enigma. J Clin Pathol. 2007;60(4):355-60.

3. Ho CM, et al. Pure-type clear cell carcinoma of the ovary as a distinct histological type and improved survival in patients treated with paclitaxel-platinum-based chemotherapy in pure-type advanced disease. Gynecol Oncol. 2004;94(1):197-203. 
4. Lee YY, et al. Prognosis of ovarian clear cell carcinoma compared to other histological subtypes: a meta-analysis. Gynecol Oncol. 2011;122(3):541-7.

5. Del CM, Birrer M, Schorge JO. Clear cell carcinoma of the ovary: a review of the literature. Gynecol Oncol. 2012;126(3):481-90.

6. Lanza G, et al. Immunohistochemical test for MLH1 and MSH2 expression predicts clinical outcome in stage II and III colorectal cancer patients. J Clin Oncol. 2006;24(15):2359-67.

7. Xicola RM, et al. Performance of different microsatellite marker panels for detection of mismatch repair-deficient colorectal tumors. J Natl Cancer Inst. 2007;99(3):244-52.

8. Allemani C. M.T.D.C., Global surveillance of trends in cancer survival 2000-14 (CONCORD-3): analysis of individual records for Global surveillance of trends in cancer survival 2000-14 (CONCORD-3): analysis of individual records for $37+\square 513+\otimes 025$ patients diagnosed with one of 18 cancers from 322 population-based registries in 71 countries. 2018. 391(17): p. 1023-1075.

9. Li GM. Mechanisms and functions of DNA mismatch repair. Cell Res. 2008;18(1):85-98.

10. Boland CR, et al. A National Cancer Institute Workshop on Microsatellite Instability for cancer detection and familial predisposition: development of international criteria for the determination of microsatellite instability in colorectal cancer. Cancer Res. 1998;58(22):5248-57.

11. Park JG, et al. Germ line mutations of mismatch repair genes in hereditary nonpolyposis colorectal cancer patients with small bowel cancer: International Society for Gastrointestinal Hereditary Tumours Collaborative Study. Clin Cancer Res. 2006;12(11 Pt 1):3389-93.

12. Peltomaki P, Vasen HF. Mutations predisposing to hereditary nonpolyposis colorectal cancer: database and results of a collaborative study. The International Collaborative Group on Hereditary Nonpolyposis Colorectal Cancer Gastroenterology. 1997;113(4):1146-58.

13. Kawakami $\mathrm{H}$, Zaanan A, Sinicrope FA. Microsatellite instability testing and its role in the management of colorectal cancer. Curr Treat Options Oncol. 2015;16(7):30.

14. Pectasides D, et al. Advanced stage clear-cell epithelial ovarian cancer: the Hellenic Cooperative Oncology Group experience. Gynecol Oncol. 2006;102(2):285-91.

15. Stelloo E, et al. Improved Risk Assessment by Integrating Molecular and Clinicopathological Factors in Early-stage Endometrial Cancer-Combined Analysis of the PORTEC Cohorts. Clin Cancer Res. 2016;22(16):4215-24.

16. Talhouk A, et al. A clinically applicable molecular-based classification for endometrial cancers. $\mathrm{Br} \mathrm{J}$ Cancer. 2015;113(2):299-310.

17. Aysal A, et al. Ovarian endometrioid adenocarcinoma: incidence and clinical significance of the morphologic and immunohistochemical markers of mismatch repair protein defects and tumor microsatellite instability. Am J Surg Pathol. 2012;36(2):163-72.

18. Xiao X, et al. Mismatch repair deficiency is associated with MSI phenotype, increased tumorinfiltrating lymphocytes and PD-L1 expression in immune cells in ovarian cancer. Gynecol Oncol. 2018;149(1):146-54. 
19. Lu Y, et al. Study of microsatellite instability in epithelial ovarian tumors. Beijing Da Xue Xue Bao Yi Xue Ban. 2006;38(1):62-5.

20. Huan Z, et al. Genetic classification of ovarian carcinoma based on microsatellite analysis: relationship to clinicopathological features and patient survival. Oncol Rep. 2008;19(3):775-81.

21. Rambau PF, et al. Significant frequency of MSH2/MSH6 abnormality in ovarian endometrioid carcinoma supports histotype-specific Lynch syndrome screening in ovarian carcinomas. Histopathology. 2016;69(2):288-97.

22. Tangir $\mathrm{J}$, et al. Frequent microsatellite instability in epithelial borderline ovarian tumors. Cancer Res. 1996;56(11):2501-5.

23. Shih YC, et al. No evidence for microsatellite instability from allelotype analysis of benign and low malignant potential ovarian neoplasms. Gynecol Oncol. 1998;69(3):210-3.

24. Arzimanoglou II, et al. Microsatellite instability differences between familial and sporadic ovarian cancers. Carcinogenesis. 1996;17(9):1799-804.

25. Cai KQ, et al. Microsatellite instability and alteration of the expression of hMLH1 and hMSH2 in ovarian clear cell carcinoma. Hum Pathol. 2004;35(5):552-9.

26. Gras E, et al. Microsatellite instability, MLH-1 promoter hypermethylation, and frameshift mutations at coding mononucleotide repeat microsatellites in ovarian tumors. Cancer. 2001;92(11):2829-36.

27. Sameer AS, Nissar S, Fatima K. Mismatch repair pathway: molecules, functions, and role in colorectal carcinogenesis. Eur J Cancer Prev. 2014;23(4):246-57.

28. Xiao X, Melton DW, Gourley C. Mismatch repair deficiency in ovarian cancer - molecular characteristics and clinical implications. Gynecol Oncol. 2014;132(2):506-12.

\section{Figures}




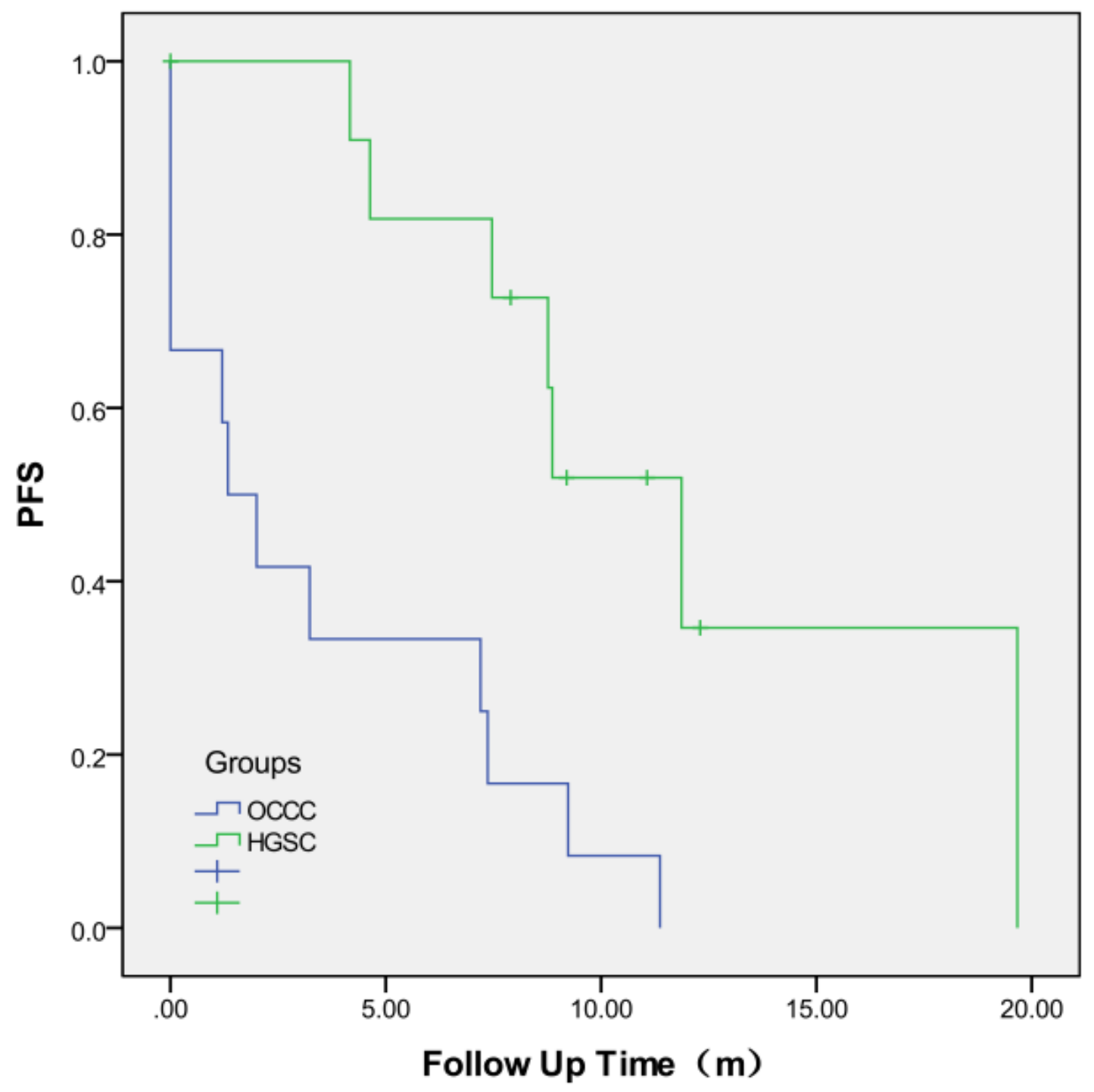

Figure 1

Kaplan Meier analysis of the PFS of patients in OCCC and HGSC groups $(P=0.001)$ 


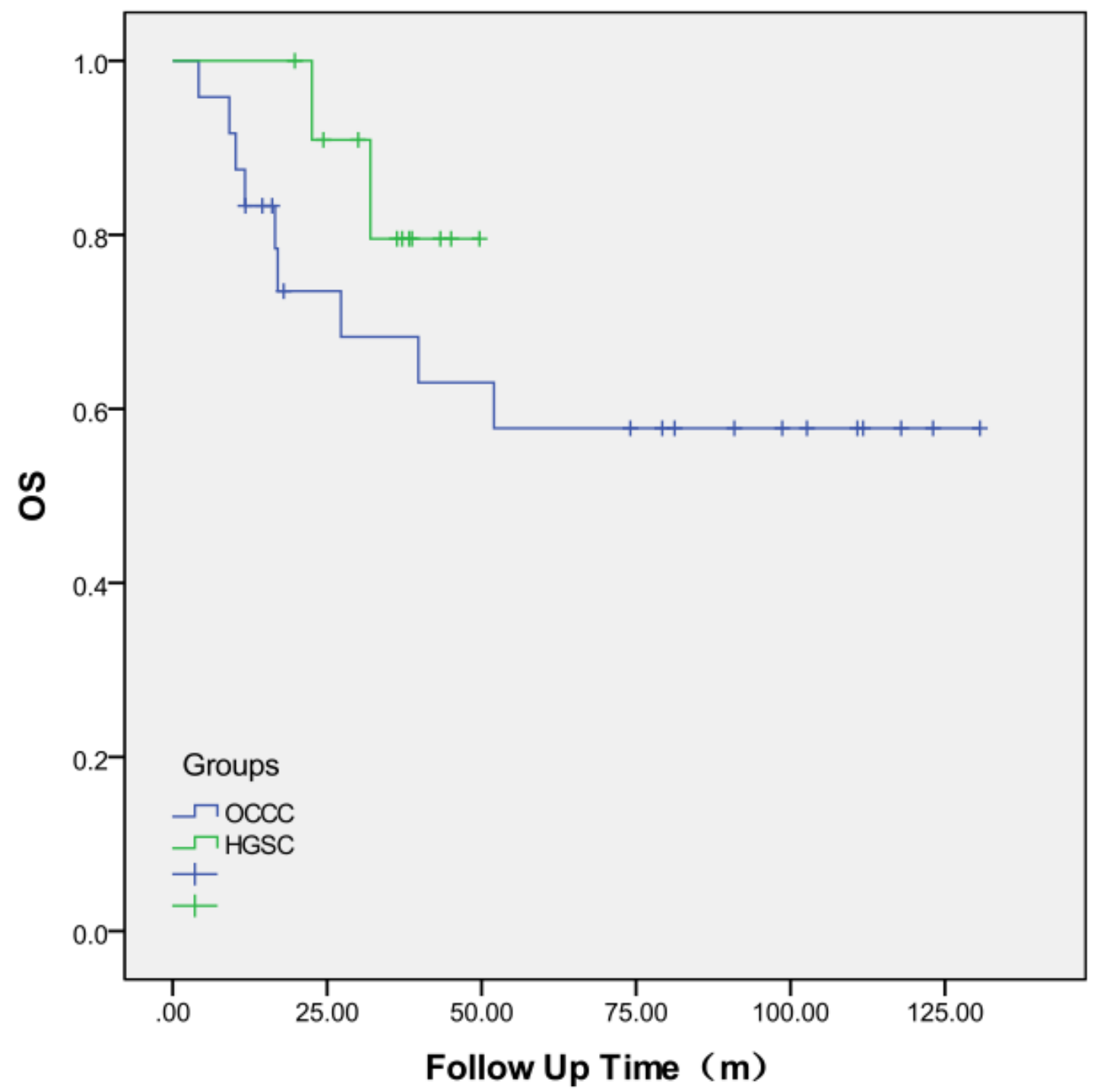

Figure 2

Kaplan Meier analysis of the OS of patients in OCCC and HGSC groups $(P=0.278)$ 


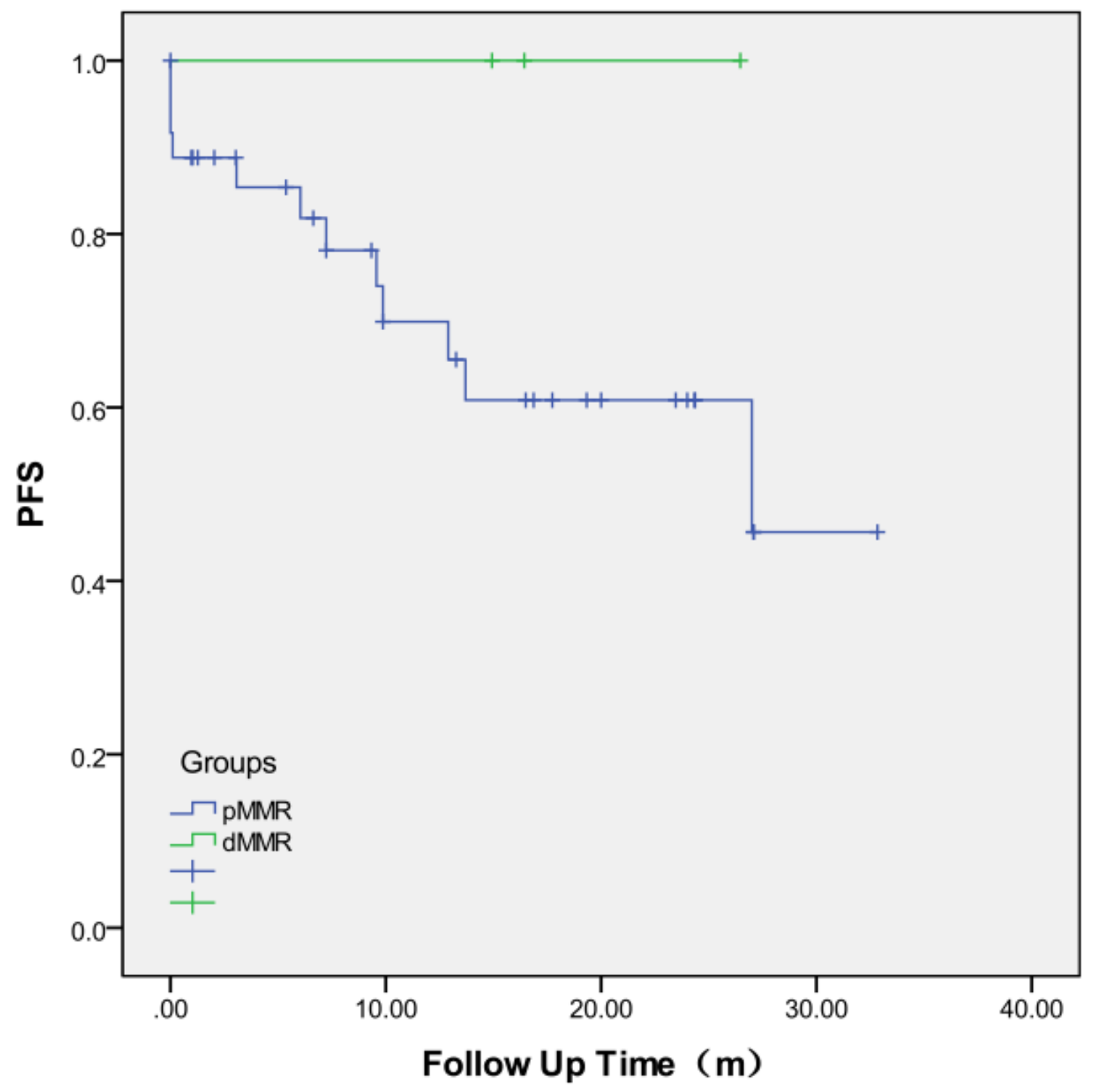

Figure 3

Kaplan Meier analysis of the PFS of patients in $\mathrm{dMMR}$ and pMMR groups $(\mathrm{P}=0.227)$ 


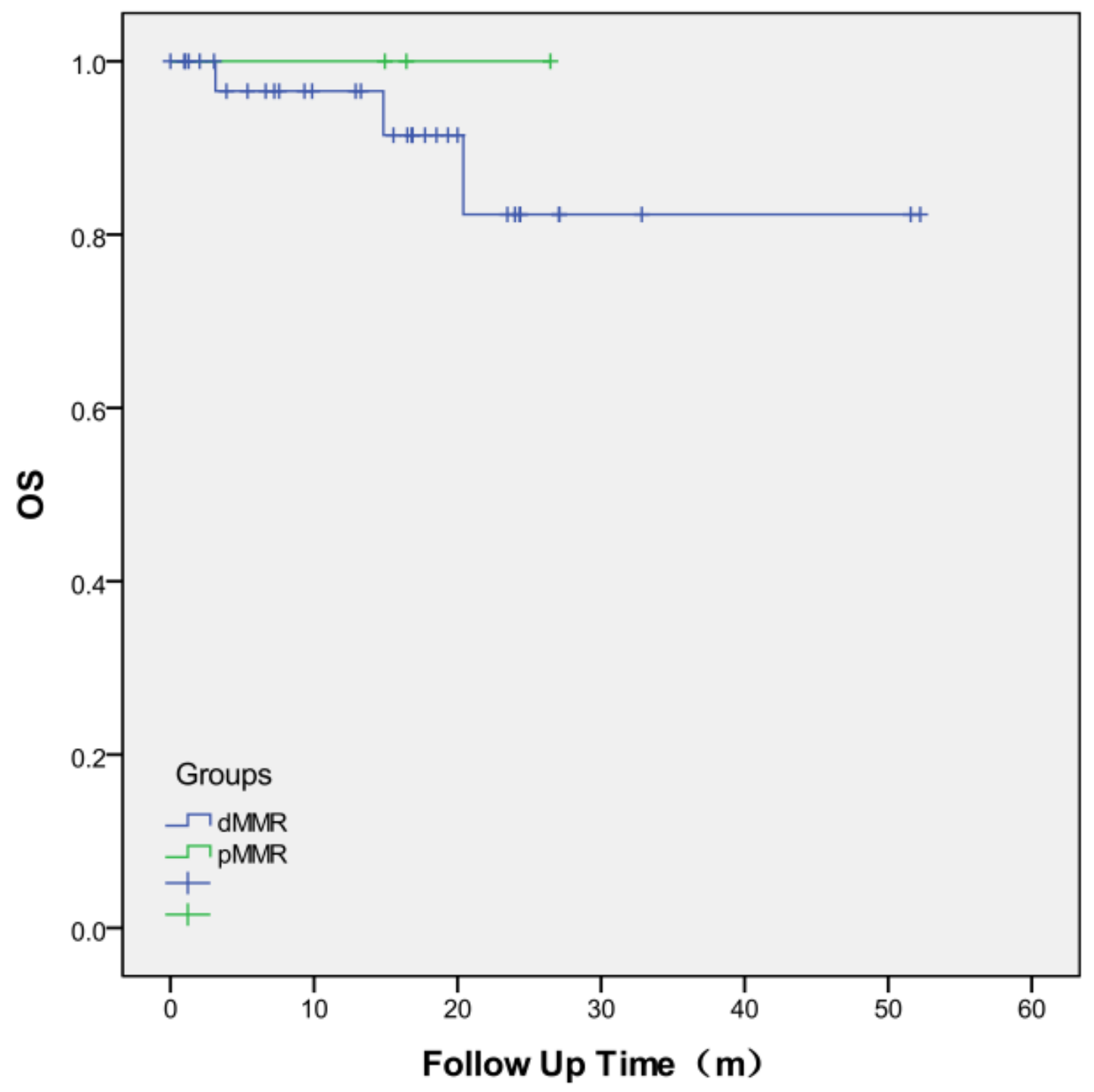

Figure 4

Kaplan Meier analysis of the OS of patients in $\mathrm{dMMR}$ and pMMR groups $(\mathrm{P}=0.548)$ 